\title{
STEM Imaging Optimized for Biological Specimen
}

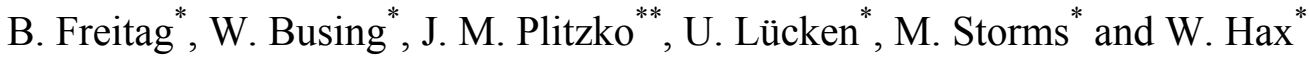 \\ * FEI Company, Achtseweg Noord 5, P.O. Box 80066, 5600 KA Eindhoven, The Netherlands \\ ** Max-Planck-Institute of Biochemistry, Molecular Structural Biology, Am Klopferspitz 18, \\ 82152 Martinsried, Germany
}

Imaging of thick, low contrast biological structures is hampered by chromatic aberration introduced by the imaging system. The problems increase whenever the sample under investigation is sensitive to the interaction with the electron beam. In order to overcome these problems, we have investigated the possible advantage of imaging biological structures in STEM mode rather than TEM mode.

Within STEM mode the signal of both the inelastic and elastic scattered electrons contribute to the contrast of the image. This is an advantage compared to zero-loss filtered TEM imaging, where a significant amount of electrons are not contributing to the image formation, especially in thicker samples, due to multiple scattering events. In combination with an ultra sensitive HAADF-detector the scattering angle can be varied to obtain the best contrast, respectively signal to noise ratio, whatever gives more flexibility to optimize the signal compared to CCD camera acquisition. With CCD cameras these parameters are fixed.

Especially, with radiation sensitive specimens it is advantageous to reduce a possible temperature increase by using a small area of illumination [1]. Thus, for a ten-fold increase in current density the temperature rise is much smaller for a focused probe than with flood-beam illumination. This is known as part of the "beam induced movement". Minimizing this effect, directly increases the dose stability of the sample in STEM compared to TEM imaging. Furthermore, as a beneficial side effect, it improves the signal-to-noise ratio (SNR) in the images [2].

The sum of these effects clearly improves the image quality of negative stained viruses and sectioned biological material, which is shown in the comparison of STEM and TEM images of biological samples from the same area (Fig. 1, 2 and 3). It is striking to see the high contrast level of biological material in the STEM images. The lower frequencies are better preserved and though this will improve and facilitate, e.g. the alignment of individual particles for single particle analysis or for the alignment of tomographic tilt series. Finally, in modern microscopes, STEM in combination with low dose acquisition schemes is already implemented [3,4], easy to use and comparable to the convenient handling of a TEM in regular bright field mode. Thus, it increases the flexibility not only in tuning and adjusting of the microscope, but also in the possibility to choose the appropriate illumination mode and camera conditions enabling the user to get the best results from investigations of biological specimens.

\section{References}

[1] L. Reimer, Springer series in optical sciences 36 (1996)

[2] P. Rez, Ultramicroscopy 96, 117-124 (2003)

[3] P. Zhang et al., Journal of Structural Biology 135, 251-261 (2001)

[4] C. Kuebel et al., Microscopy \& Microanalysis 8, 792-793 (2002)

[5] U. Ziese et al., Journal of Structural Biology 138, 58-62 (2002) 

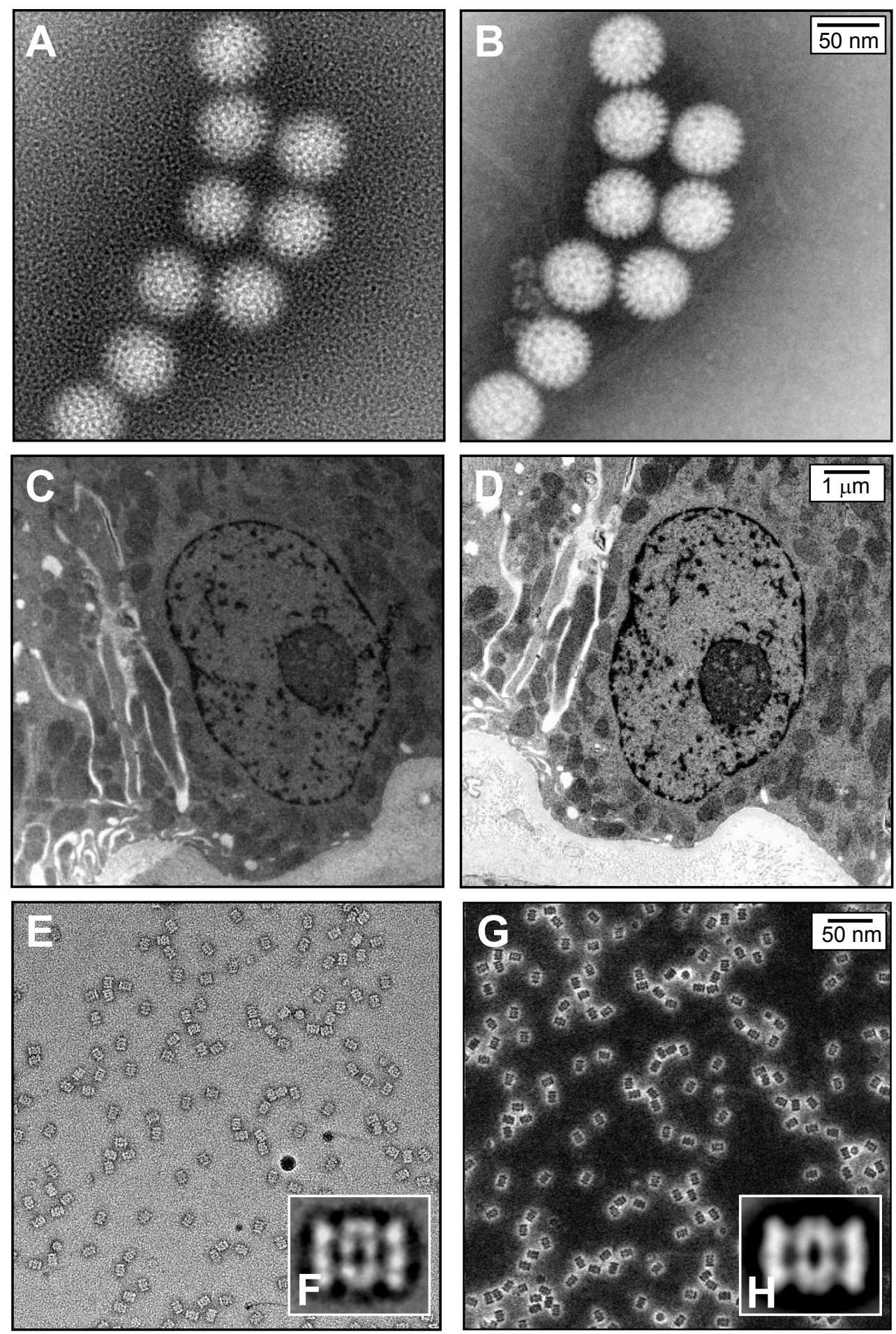

Figure 1: (A) Zero-loss filtered TEM image of a renal biopsy section and (B) contrast inverted HAADF-STEM image of the same sample region. (C) Zero loss filtered TEM image of a negatively stained adnovirus and (D) contrast inverted HAADF STEM image of the same sample area. (E) Bright field image of negatively stained proteasome 20S molecules. (F) 2-dimensional averaged image from 97 particles. (G) STEM image of the same sample area and (H) 2-dimensional averaged image from 97 particles. 